\title{
Magnetopause current as seen by Cluster
}

\author{
M. W. Dunlop ${ }^{1,2}$ and A. Balogh ${ }^{2}$ \\ ${ }^{1}$ SSTD, Rutherford Appleton Laboratory, Chilton, Didcot, Oxon, OX11 0QX, UK \\ ${ }^{2}$ Imperial College, Exhibition Road, London SW7 2BW, UK
}

Received: 26 May 2004 - Revised: 17 January 2005 - Accepted: 21 January 2005 - Published: 30 March 2005

\begin{abstract}
The four-spacecraft, magnetic field measurements on Cluster can be combined to produce an accurate determination of the electric current in the magnetopause boundary during stable magnetopause crossings. For events that are planar on the scale of the spacecraft configuration, the thickness of the current layer can be accurately estimated from its magnetic profile at each spacecraft and the corresponding boundary crossing times. The latter, give a determination of boundary motion relative to the Cluster array. We use the estimates of all these properties, for a range of spacecraft separation distances, to show, firstly, that the estimate of electric current density is representative even when the spatial scale of the configuration of Cluster spacecraft approaches the thickness of the current layer. Secondly, we show that the estimated current lies in the plane of the boundary and demonstrate this for crossings occurring during large-scale ripples on the magnetopause. Thirdly, we show that the magnitude of the current is accurately represented, averaged over the extent of the current layer, by comparing to the change in the boundary-parallel magnetic field component divided by the estimated current layer thickness. We demonstrate this last point using a range of crossings each having a different thickness and crossing speed, different changes in the magnetic field component and different current densities.
\end{abstract}

Keywords. Magnetospheric physics (Current systems; Magnetopause, cusp and boundary layers)

\section{Introduction}

Although the magnetopause has been extensively studied for many years (e.g. Berchem and Russell, 1982; Russell, 1995; Paschmann et al., 1985, 1986; Phan et al., 1994; Phan and Paschmann, 1995), direct measurement of the vector current density has not been possible until the advent of the four spacecraft Cluster mission (Space science reviews, 1997). Previous multi-point observations of the magnetopause layer have primarily arisen from dual spacecraft: ISEE1 and 2 (Russell and Elphic, 1978, 1979) and AMPTE IRM and UKS (Bryant et al., 1985) measurements, e.g. Elphic, et al. (1988,
1990, 1995), although estimates of the current density has been attempted in the past, e.g. Van Allen and Adnan (1992). The spatial array of Cluster spacecraft also allows the local geometry of the magnetopause to be determined to a large degree and so provides a direct comparison of the current within the boundary layer as it is traversed. The four Cluster spacecraft were launched in pairs on two Soyuz rockets on the 16 July and the 9 August 2000 and were placed into eccentric $\left(4 R_{E} \times 19.6 R_{E}\right)$, inertial, polar orbits. Throughout a year, the orbit covers the dayside magnetopause at high latitude near local noon and low latitude near the dawn and dusk flanks. The spacecraft fly in an evolving configuration, which repeats every orbit (apart from minor perturbations), but which has been changed at intervals during the mission to cover a large range of spacecraft separation distances (100$6000 \mathrm{~km}$ ) at the magnetopause. The results presented here, therefore, have been confirmed over a variety of spatial scales and for different locations on the magnetopause.

Individual spacecraft crossing the magnetopause sample changes in the magnetic field across the current layer, which, in a 1-D geometry, can in principle give an estimate of the current magnitude in the boundary. These estimates, however, depend on accurate knowledge of the orientation and motion of the boundary, in order to obtain positions within the layer. A number of single spacecraft techniques exist to estimate boundary orientation and motion, but the four-spacecraft Cluster magnetic field measurements (Balogh et al. 2001) alone have allowed direct determination of the boundary motion, giving accurate measurement of the boundary thickness and orientation (Dunlop et al. 2002a). The local current density (Dunlop et al. 2002b) is also estimated from the four spacecraft magnetic field measurements alone and we directly compare these quantities for a number of events covering different spatial scales and different geometrical circumstances. A number of related four spacecraft techniques now exist (e.g. Haarland et al. 2003), making either a variety of assumptions or using other measurements, which can provide additional confirmation of the results or improvement in accuracy in certain circumstances. Here we intend to demonstrate the key quantitative result uniquely determined from Cluster magnetic field measurements.

Correspondence to: M. W. Dunlop

(m.dunlop@rl.ac.uk) 
Initially we briefly describe below the magnetic field experiment and the key data analysis methods used and then discuss the results for selected event examples. The intention in this brief report is to show how rugged are the basic methods (rather than presenting comparative analysis of the four spacecraft data); particularly with the virtue of using only one data set having extremely high accuracy, namely the magnetic field, supported by the high accuracy of the auxiliary position data.

\section{Measurements and techniques}

The fluxgate magnetometer (FGM) experiment provides high time resolution magnetic field measurements on all four spacecraft (Balogh et al., 2001) at $22.4 \mathrm{~Hz}$ (normal mode) or $67 \mathrm{~Hz}$ (burst mode). The instruments are operating continuously and the data have been filtered and re-sampled onboard from an internal sampling rate of $202 \mathrm{~Hz}$. The data are believed to be inter-calibrated to at least $0.1 \mathrm{nT}$ accuracy overall. Here, we employ both spin resolution and high time resolution data where appropriate, which has been re-calibrated to higher accuracy where necessary. The time series data shown in the plots is at spin resolution.

The discontinuity analyser (DA) technique determines parameters that describe the motion, geometry and orientation of discontinuities. The basic algorithm determines boundary normals at each spacecraft crossing point, independently (using Minimum Variance Analysis (Sonnerup and Cahill, 1967), for example). It is applied here in the case of stationary, planar boundaries, where the normals are all found to be parallel. The boundary orientation and motion can then be calculated by combining the boundary crossing times with the spacecraft separation vectors. Because the normals are determined independently, parallel normals not only imply a planar geometry over the spacecraft array, but provide mutual confirmation of the boundary orientation (mean normal), which then allows the boundary motion $\left(v_{n}, a_{n}\right)$, to be determined from

$r_{n}=v_{n}^{0} t+1 / 2 a_{n} t^{2}$

where $\boldsymbol{r}_{n}=\Delta \mathbf{r}_{i j} \cdot \mathbf{n}, t=t_{i j}$, etc. and $i, j$ refer to the spacecraft.

If the motion is constant ( $a_{n}$ approximately zero), Eq. 1 can be used to compute $\mathbf{n} / v_{n}^{0}$ (after Russell et al. 1983). The computation of $\mathbf{n}$ and $v_{n}^{0}$, made by assuming constant velocity, is referred to as a timing analysis, since it only depends on the timing of the crossings at each spacecraft. In practice, application requires identifiable, stationary features in the time series data, usually best viewed in the maximum variance component of the magnetic field. For the case of well-defined current sheets, this component will suggest a magnetic field rotation over some finite time interval (traversal time to cross the current sheet). Once any change in velocity has been found, each traversal time through the current layer can be scaled to a boundary thickness (at each spacecraft).
The first application of the curlometer technique has been reported by Dunlop et al. (2002b). The method combines simultaneous, vector magnetic field data from each spacecraft with the spacecraft positions to calculate the curl of the magnetic field from Ampère's law (giving an estimate of the average current density through the spacecraft configuration), using the difference approximation

$\mu_{0} \boldsymbol{J} \cdot\left(\Delta \boldsymbol{r}_{i} \wedge \Delta \boldsymbol{r}_{j}\right)=\Delta \boldsymbol{B}_{i} \cdot \Delta \boldsymbol{r}_{j}-\Delta \boldsymbol{B}_{j} \cdot \Delta \boldsymbol{r}_{i}$ $\left\{\right.$ representing $\left.: \mu_{0} \int \boldsymbol{J} \cdot d s=\oint \boldsymbol{B} \cdot d \boldsymbol{l}\right\}$

with $\Delta \boldsymbol{r}_{i} \equiv \boldsymbol{r}_{i}-\boldsymbol{r}_{1}$, and similarly $\Delta \boldsymbol{B}_{i} \equiv \boldsymbol{B}_{i}-\boldsymbol{B}_{1}$, giving the average current normal to each face $(1, i, j)$ of the spacecraft tetrahedron. Since each face is known by $\Delta \boldsymbol{r}_{i \wedge} \Delta \boldsymbol{r}_{j}$, the currents normal to three faces can be re-projected into a Cartesian co-ordinate system. It is also possible to calculate an estimate for $\operatorname{div}(\boldsymbol{B})$, from

$\operatorname{div}(\boldsymbol{B})\left|\Delta \boldsymbol{r}_{i} \cdot \Delta \boldsymbol{r}_{j \wedge} \Delta \boldsymbol{r}_{k}\right|=\left|\sum_{\text {cyclic }} \Delta \boldsymbol{B}_{i} \cdot \Delta \boldsymbol{r}_{j \wedge} \Delta \boldsymbol{r}_{k}\right|$,

which produces non zero values, partially as a consequence of non-linear spatial gradients neglected in its estimate. This quantity therefore usefully measures an effect of the linear approximation and we use it as a monitor of this error.

\section{Results}

\subsection{Establishing the MP current}

We first demonstrate that the curlometer technique is able to consistently estimate the magnetopause current using an example taken from 26 January 2001. The left-hand panel in Fig. 1 shows the spacecraft configurations (enlarged by a factor of 20) along the orbit, which correspond to an outbound pass through the dusk-side magnetopause, crossing at high $\left(\sim 9.5 \mathrm{R}_{E}\right)$ latitude, as shown. In this Figure (and the others showing the orbit track) the dots along the orbit are at hour intervals. The mean spacecraft separation distance was $600 \mathrm{~km}$. These data have been reported by Bosqued et al. (2001), Pu et al. (2002) and Phan et al. (2003) and exhibit a key interval of repeated boundary layer crossings as a result of inward and outward motion of the magnetopause between 10:00 and 11:00 UT (which has a mean thickness of $\sim 1200$ $\mathrm{km}$ and mean speed of $\sim 25 \mathrm{~km} / \mathrm{s}$ ). The right hand plot of Fig. 1 shows a short segment of the orbit, projected into $X, Y_{G S M}$, with cuts through a model magnetopause (Sibeck et al., 1991), which are placed at 10:00 and 11:00 UT so as to represent the approximate range of positions along the orbit at which the boundary is sampled. The components of the estimated current density are plotted along the orbit (in $\mathrm{nAm}^{-2}$, scaled down by a factor of 10) and show enhanced values within this interval and a clear alignment to the magnetopause orientation. Between 10:00 and 11:00 UT the current bursts correspond to the repeated encounters with 


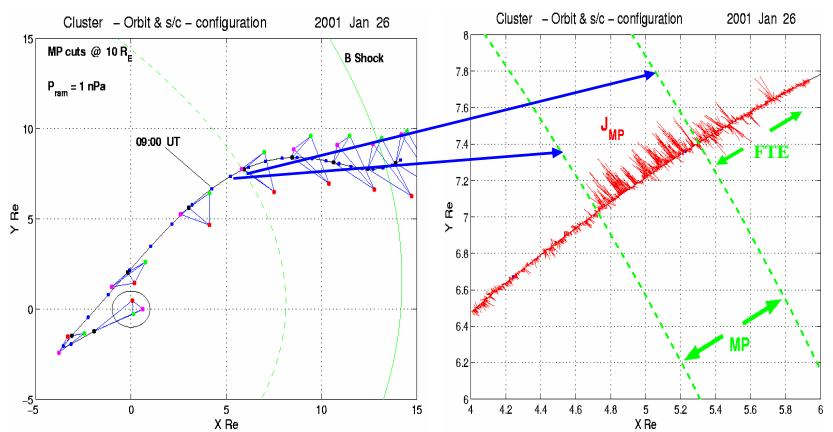

(a)

(b)

Fig. 1. The analysis of the 26 January 2001. (a) The Cluster orbit track, projected into the GSM X,Y plane, together with the configuration of the four spacecraft, enlarged by a factor of 20 at intervals along the orbit. Magnetopause and bow shock curves are shown at the high of the orbit track and for the observed solar wind pressure, as indicated. (b) The computed current estimate from the curlometer, projected into the plane along the orbit track (given in $\mathrm{nAm}^{-2}$, scaled down by a factor of 10). The dashed lines indicate the extreme positions on the orbit within which the magnetopause is encountered. The magnetopause moves inward and outward several times during the interval, resulting in the bursts of current which are apparent from the projections.

the magnetopause boundary layer, and are directed predominantly in one direction, consistent with a Chapman/Ferraro current.

The mean current for this interval is: $(-7.4,8.4$, $-0.65)_{G S M}, \mathrm{nAm}^{-2}$. The overall variance in this mean current direction for all the crossings is $\sim 14 \mathrm{deg}$. When the variation of the current vector is calculated only over the period of each crossing for which the magnitude is more than $60 \%$ of the peak current the vectors are aligned to $<5 \mathrm{deg}$. More detailed analysis of the individual crossings (not included here for this event, but see below for other passes) shows that the slight tilting of the current directions from crossing to crossing are consistent with corresponding tilting of the local magnetopause direction, which then accounts for the size of the variance calculated above. In contrast, enhanced values of current after 11:00 UT, correspond to a train of FTE (flux transfer event) signatures, associated with the occurrence of extensive reconnection during the event (Phan et al., 2003) and exhibit a variety of orientations. The mean current for this later interval is: $(0.06,-0.07,-0.9)_{G S M}, \mathrm{nAm}^{-2}$, which is an order of magnitude lower and directed along $\mathrm{Z}_{G S M}$ (the mean flux tube direction), rather than the plane of the magnetopause.

\subsection{Orientation to MP ripples}

Figure 2 shows a second event, which occurred as the spacecraft were at apogee in the dawn flank magnetosheath, near the equatorial plane. Figure $2 b$ shows a schematic of the boundary motion, superimposed onto the orbit which is shown projected into the $\mathrm{X}, \mathrm{Y}_{G S M}$ plane and in the same format as Fig. 1. In this case the spacecraft configurations have

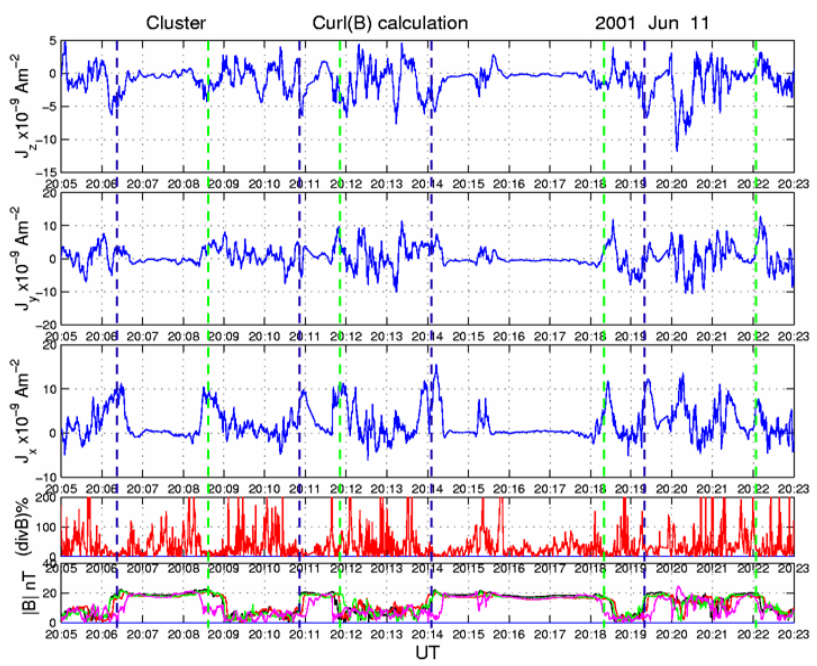

(a)

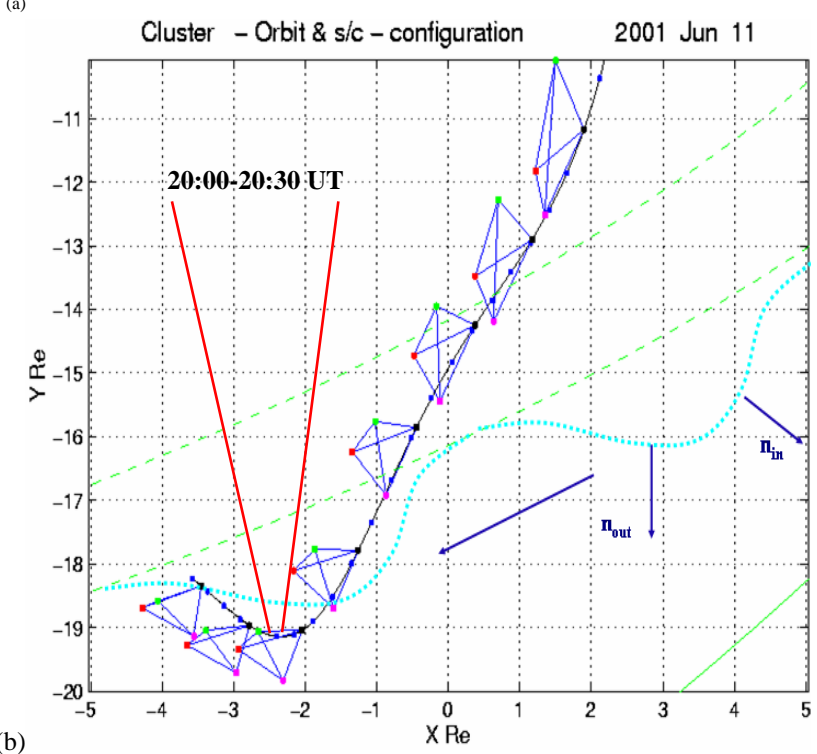

Fig. 2. The analysis of the 11 June 2001, showing (a) a plot of the curlometer estimate of GSM components of the current (top three panels), the estimate of $\operatorname{div}(\mathrm{B})$ and the total magnetic field from all four spacecraft to show the pairs of crossings to and from the magnetosphere, and (b) the orbit track and spacecraft configuration projected into GSM together with a schematic of the boundary ripples implied from the DA analysis.

been scaled by a factor of 5 and the mean separation distance is $\sim 2000 \mathrm{~km}$. The DA analysis suggests that a series of surface ripples on the magnetopause move tailward past the spacecraft array in the manner indicated, taking them fully into the magnetosphere on each occasion. The lower panel in Fig. 2a shows the multi-spacecraft, magnetic field magnitude where the transitions from a quiet, $20 \mathrm{nT}$ magnetospheric field to a low, fluctuating magnetosheath field are marked by the dashed lines. The magnetopause orientations fall into pairs for inward/outward crossings, represented by the green and blue vertical dashed lines on the plot in Fig. 2a, respectively, and match the two distinct boundary orientations 


\begin{tabular}{|c|c|c|c|c|c|c|c|c|c|c|c|c|c|c|c|c|c|c|c|}
\hline \multirow{2}{*}{$\begin{array}{c}\text { Date } \\
11 / 06 / 2001\end{array}$} & \multirow{2}{*}{$\frac{\text { UT }}{20: 06: 30}$} & \multirow{2}{*}{$\frac{\mathrm{MP}}{1 \text {-out }}$} & \multirow{2}{*}{$\frac{\left|\lambda_{2} / \lambda_{3}\right|}{\mid 15.3}$} & \multicolumn{2}{|c|}{$\left\langle\mathrm{n}_{\mathrm{GSE}}\right\rangle$} & \multirow{2}{*}{$\frac{\left\langle\mathrm{V}_{\mathrm{n}}\right\rangle}{120}$} & \multicolumn{3}{|c|}{$\mathrm{n}_{\text {timing }}$} & \multirow{2}{*}{\begin{tabular}{r|}
$\mathrm{V}_{\text {timing }}$ \\
61
\end{tabular}} & \multicolumn{3}{|c|}{$\mathrm{J}_{\mathrm{GS}}$} & \multirow{2}{*}{$\frac{|\mathrm{J}|}{10}$} & \multirow{2}{*}{\begin{tabular}{r|}
$\Delta \mathrm{B}$ \\
18
\end{tabular}} & \multirow{2}{*}{$\frac{\Delta \mathrm{D}_{1}}{1214}$} & \multirow{2}{*}{$\frac{\Delta \mathrm{D}_{2}}{1244}$} & \multirow{2}{*}{$\frac{\Delta \mathrm{D}_{3}}{1214}$} & \multirow{2}{*}{$\frac{\Delta \mathrm{D}_{4}}{1171}$} \\
\hline & & & & $\begin{array}{ll}0.27 & -0.94\end{array}$ & 0.19 & & 0.03 & -0.83 & 0.56 & & 8 & 3 & -4 & & & & & & \\
\hline & & 1 -in & 9.0 & $0.70-0.62$ & -0.36 & -104 & 0.22 & -0.95 & 0.22 & -45 & 8 & 6 & -2 & 10 & 19 & 1297 & 1380 & 1331 & 1438 \\
\hline & $20: 11: 00$ & 2-out & 5.0 & $0.46-0.88$ & 0.13 & 155 & -0.16 & -0.82 & 0.55 & 106 & 8 & 3 & -4 & 10 & 19 & 1514 & 1492 & 1420 & 1448 \\
\hline & & 2-in & 5.7 & $\begin{array}{ll}0.66 & -0.73\end{array}$ & 0.21 & -74 & 0.68 & -0.48 & 0.55 & -106 & 9 & 7 & -4 & 11 & 23 & 1088 & 1031 & 1154 & 1120 \\
\hline & $20: 14: 00$ & 3-out & 7.7 & $\begin{array}{ll}0.07 & -0.99\end{array}$ & 0.15 & 32 & 0.34 & -0.94 & 0.04 & 71 & 11 & 4 & -5 & 13 & 25 & 1228 & 1545 & 1412 & 1358 \\
\hline 05/07/2001 & $05: 24: 00$ & $\mathrm{mp}$ & 11.0 & $0.53-0.84$ & -0.11 & -111 & 0.41 & -0.81 & -0.41 & -89 & 5 & -20 & -15 & 27 & 59 & 751 & 595 & 551 & 710 \\
\hline & $05: 35: 00$ & $\mathrm{mp}$ & 3.0 & $\begin{array}{lll}0.36 & -0.84\end{array}$ & 0.41 & 20 & 0.43 & -0.87 & 0.25 & 17 & 24 & -26 & -18 & 40 & 55 & 274 & 291 & 299 & 318 \\
\hline & $05: 45: 00$ & $\mathrm{mp}$ & 6.0 & $0.64-0.76$ & -0.11 & -22 & 0.53 & -0.82 & 0.19 & -44 & 14 & -30 & -25 & 40 & 63 & 450 & 391 & 415 & 481 \\
\hline & 06:06:00 & $\mathrm{mp}$ & 7.8 & $0.28-0.85$ & 0.44 & 11 & 0.24 & -0.82 & 0.52 & 11 & 22 & -22 & -15 & 35 & 51 & 344 & 356 & 367 & 350 \\
\hline & $06: 23: 00$ & $\mathrm{mp}$ & 3.7 & $0.61-0.78$ & -0.03 & -23 & 0.54 & -0.83 & 0.17 & -41 & 13 & -17 & -16 & 25 & 53 & 836 & 761 & 761 & 867 \\
\hline
\end{tabular}

Table 1. Summary of the boundary analysis for crossings from two days, giving the boundary normals (n) and speed from both the DA and timing analysis, the curlometer (current in $\mathrm{nAm}^{-2}$ ) and the thickness $(\Delta \boldsymbol{D}$ in $\mathrm{km}$ ) for each event. Magnetic field is given in $\mathrm{nT}$ and velocities in $\mathrm{km} / \mathrm{s}$. The boundary normals are unit vectors.

"in" and "out" refer to entry and exit from the cusp region respectively. $V_{n}$ refers to component a long the normal directions quoted.

shown in Fig. 2b. The boundary normals are depicted by the blue arrows shown in Fig. $2 b$ (which represent their mean orientations) and have small $\mathrm{Z}_{G S M}$ components. The crossings are irregular and show a different velocity projection, at each spacecraft, along the boundary normals for the crossings. This motion together with transit times through each current layer encounter (measured from the turning in the maximum variance component of the field) can be used to estimate boundary thickness (see below in Table 1), but represents a projected tailward motion along the magnetopause for each crossing. The value of divB is also included in Fig. $2 \mathrm{a}$ to show that the value is low $(\sim 15 \%)$ at each crossing.

The other panels in Fig. $2 \mathrm{~b}$ from bottom to top show respectively the estimate of $\operatorname{div}(\mathrm{B})$ and the GSM components of curl(B), $\mathrm{J}_{X, Y, Z}$. Although the spacecraft separation distances are slightly larger than the estimates of boundary thickness (see Table 1), it appears that the current density is still adequately sampled through each crossing to vindicate its direction. In fact, the direction of the current maintains its alignment to the magnetopause orientation as it tilts from crossing to crossing. This can be seen from the components of curl(B), which change from crossing to crossing. The $\mathrm{J}_{X}$ component is maintained for the majority of crossings, whereas the $\mathrm{J}_{Y}$ component changes so that the majority of the inward crossings (green dashed lines, taking the spacecraft back into the magnetosheath) have larger values than the outward crossings, which tend to have small $\mathrm{J}_{Y}$ components. This follows the orientations indicated for the geometry of the ripples (as shown by the arrows in Fig. 2a). The $\mathrm{J}_{Z}$ component is less significant for magnetopause alignment since the normals lie nearly in the $\mathrm{X}, \mathrm{Y}_{G S M}$ plane. Thus, even for this event, where the magnetopause current layer is poorly matched in scale to the spacecraft separation, the curlometer is giving a good estimate of direction.

\subsection{Scaling of the current density}

Figure 3 shows a further series of magnetopause crossings from a third event. The Cluster orbit skirted the dawn flank magnetopause during this day, maintaining a position relative to the boundary, which moved from high to low-latitudes (as shown in Fig. 3a). This location provided a number of magnetopause crossings for a large fraction of the day and the interval shown in Fig. $3 b$ represents a few crossings from this set. The figure shows the magnetic field in the top four panels and the curlometer estimate in the bottom three, all in GSM. Distinct from the previous event and given the spacecraft separations and estimated motion determined from the DA technique, these crossings represent small amplitude $\left(<1 \mathrm{R}_{E}\right)$, inward and outward motions of the magnetopause, providing a sequence of crossings with almost parallel orientations. The bursts of current in the lower three panels reflect this common orientation by maintaining a common direction, aligned to the magnetopause plane. It should be noted however, that in this event the scale size of the boundary layer is smaller compared to the cluster separation distance (see below) and we expect a less close quantitative comparison between the current magnitude and direction. The current signature shows both slow crossings, where the spacecraft array remains in the current layer for longer so that the enhanced value of curl(B) remains for minutes, and fast crossings, where the curl(B) profile shows a short spike.

These slow and fast crossings are listed in Table 1, as indicated by their mean velocities. Table 1 quantifies the results and summarises both the last event and a selected set of crossings from 5 July 2001, which have been analysed in detail, and shows the estimated thickness of the current layer for each crossing, together with the current density estimates. The columns show respectively the event times, the crossing label (referring to "in" and "out" for the dashed lines in Fig. 2 and to "mp" for the dashed lines in Fig. 3). From left to right the remaining columns show: the mean MVA normal and velocity (in $\mathrm{km} / \mathrm{s}$ ) from the DA technique; the corresponding timing normal and velocity (in $\mathrm{km} / \mathrm{s}$ ); the average current across the current layer, estimated from the curlometer (in $\mathrm{nA} / \mathrm{m}^{2}$ ); the change in the maximum variance component of the magnetic field across the magnetopause (in nT), and the 


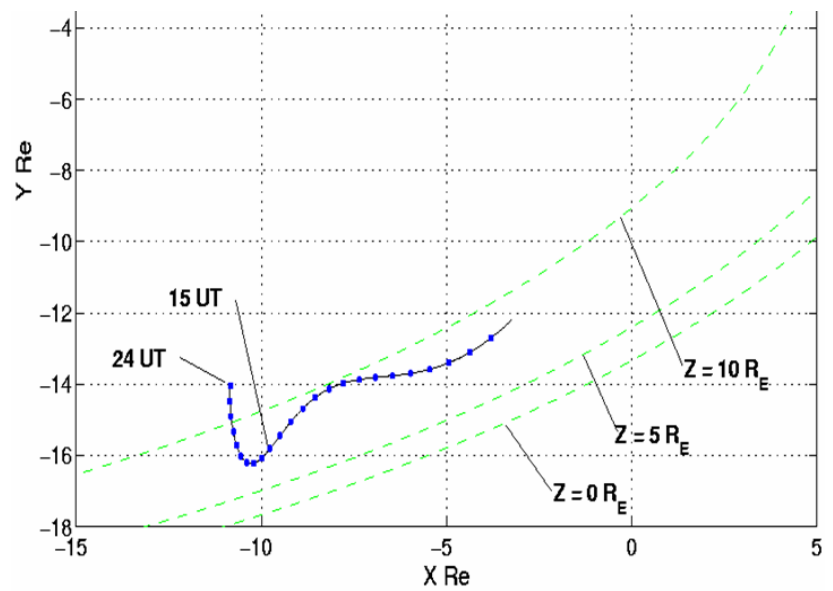

(a)

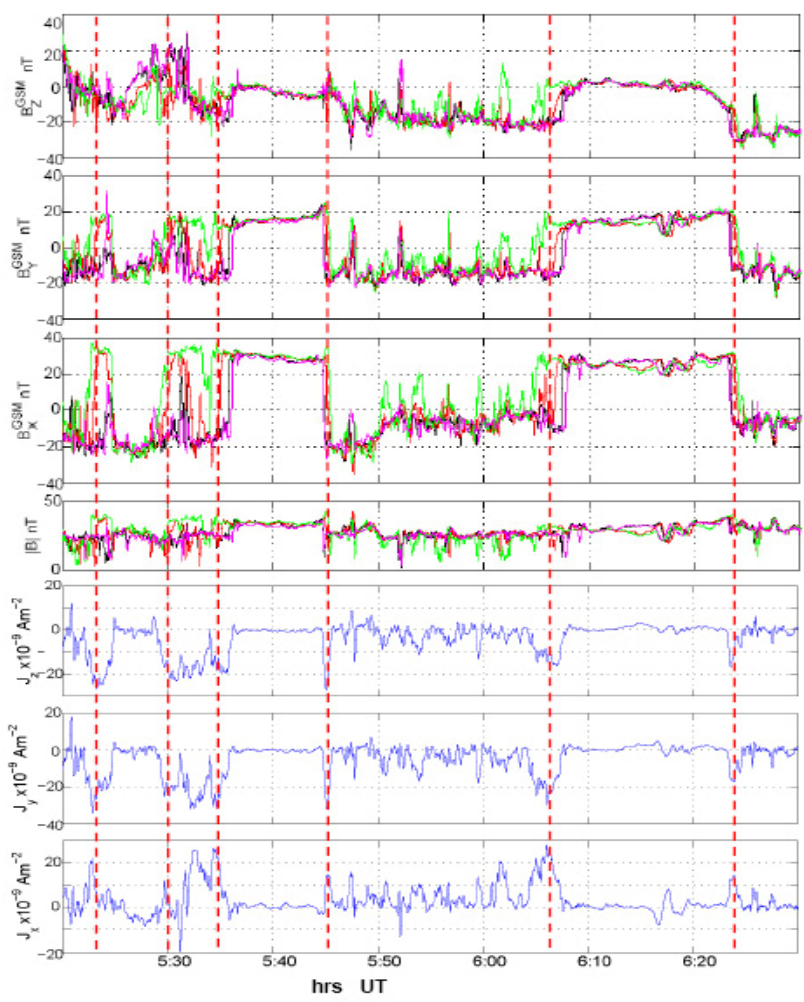

(b)

Fig. 3. The analysis of the 5 July 2001. (a) The orbit track projected into GSM for the whole day, together with cuts through the magnetopause at the heights and solar wind pressure indicated. (b) A plot of the four spacecraft magnetic field data (top four panels) and the GSM components of the current estimate (lower three panels), for a short interval containing a few of the magnetopause crossings observed on this day.

estimated thickness of the current layer at each spacecraft from the DA technique (in $\mathrm{km}$ ). The timing normals, shown for comparison, assume constant velocity, which is often not true for these crossings. Consequently, they highlight the sensitivity in the DA analysis to compute boundary motions, and therefore the scaling of the current thicknesses. These

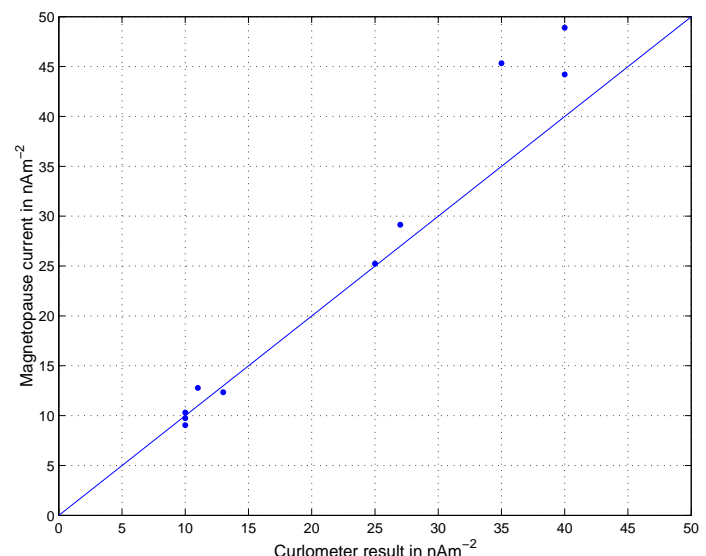

Fig. 4. A scatter plot of the results in the table, showing the estimated magnetopause current $(\Delta \boldsymbol{B} / \Delta \boldsymbol{D})$ against the curlometer calculation $(J)$.

estimates, however, show good stability for all the crossings shown.

The results confirm that the thickness of the current layer is effectively constant while being sampled by the spacecraft array, the small variations being within the estimated errors. This thickness can be used to compute an average current density in the magnetopause from $\Delta \boldsymbol{B} / \Delta \boldsymbol{D}$, where $\Delta \boldsymbol{B}$ and $\Delta \boldsymbol{D}$ are taken from Table 1. This can then be compared to $\mu_{0}|\mathrm{~J}|$. For each crossing, the variation of the current follows this ratio within $15 \%$ uncertainty (worst case). Moreover, the estimate of the current vector lies nearly perpendicular to the DA-normals also shown in the table and therefore lie parallel to the magnetopause boundary, to within the same uncertainty. The tilting of the boundary ripples in the first event, in particular, can also be seen in both the current vector and the DA-normals. We do not expect such close quantitative agreement in the case of the second event where the relative scale size of the boundary is smaller (thinner), but although the errors appear to be larger, there is evidence that the scaling of the curlometer current follows that of $\Delta \boldsymbol{B} / \Delta \boldsymbol{D}$ as before. These quantitative results can be read off the profiles observed in Fig. 3, in particular, where the $\Delta \boldsymbol{B}$ clearly changes from crossing to crossing, reflecting, mainly, the changing $\Delta \boldsymbol{D}$. The mean currents for each of the crossings indicated by the dashed lines are given in Table 1 . The variance in these currents is $<0 \mathrm{deg}$, reflecting the less accurate calculation of the curlometer in this case. Figure 4 shows a scatter plot of the results given in Table 1, and compares the two estimates of current from the curlometer and $\Delta \boldsymbol{B} / \Delta \boldsymbol{D}$ (magnetopause current), with appropriate scaling for the thickness estimates. The values of magnetopause current for the higher current values can be seen to be slightly over estimated.

\section{Conclusions}

We have shown that the magnetic field measurements on Cluster can produce a realistic determination of the electric 
current density at the magnetopause, even where the scale size of the Cluster configuration approaches that of the current layer thickness, using a series of planar magnetopause crossings with significant shear. The vector current densities have been shown to lie in the plane of the magnetopause boundary (to within 15\%), even during times of induced motion and large-scale surface ripples. This is the first time that in situ measurements have directly measured the magnetopause current. These results have depended upon first accurately determining the orientation and thickness of the current layer, using the four spacecraft DA technique. In combination with the curlometer estimate of $\operatorname{curl}(\mathrm{B})$, the current profiles have been shown to compare well to the mean current defined by the overall magnetic shear across the boundary layer. The thicknesses determined here, for a number of crossings from the three passes, vary between 300$1500 \mathrm{~km}$ (see Table 1), which is comparable, for each pass, to the estimated range of ion gyroradii. The corresponding current densities range from $\sim 9 \mathrm{nAm}^{-2}$, for the thickest boundaries, to $\sim 40 \mathrm{nAm}^{-2}$, for the thinnest. The magnetopause crossing speeds (not shown here) ranged from $\sim 10-30 \mathrm{~km} / \mathrm{s}$ (at the location near local noon) to $\sim 100-150 \mathrm{~km} / \mathrm{s}$ (at the dawn flank location).

Knowledge of the boundary orientation and motion in principle allows a mapping of time to spatial location in the current layer (through: $\delta \mathrm{x}=\mathrm{v}_{n} \delta$ t, if $x$ lies along the boundary normal). This allows the current density to be compared to the magnetic field change with distance through the layer. For example, the current estimates shown in Figs. 1-3 all show simple peak-profiles though each encounter with the magnetopause. Some correspond to fast (sharp) crossings and some to slow encounters. Given the velocity estimates from the DA technique at each spacecraft $\left(v_{n}\right)$, the change in the magnetic field component with time through the magnetopause can therefore be related to distance through the boundary layer, and hence to the current profile (since: $v_{n} \mu_{0}|\mathrm{~J}|=\delta \mathrm{B} / \delta \mathrm{t}$ ). This information is limited, of course by the relative scale size of the Cluster array and changing velocity at each spacecraft, since the curlometer gives a mean current estimate over the whole spacecraft volume. Future work is underway which explores the use of the average field over the four spacecraft in this context and using selected crossings at small spacecraft separations and constant boundary motion.

It is also the subject of further studies to investigate, using these tools, the dependence of the current layer on both magnetopause location and external conditions in the adjacent magnetosheath. For example, one event here (26 January 2001) has corresponded to a very active period of reconnection (Phan et al. 2003) with an established plasma boundary layer, whereas the other events do not. The two flank events (11 June 2001 and 5 July 2001) are in contrast since in the first case the magnetopause thickness appears to be relatively stable from crossing to crossing, whereas in the second it varies. Moreover, in the first event, the electron and ion data (not shown here) indicate that a boundary layer appears to be absent.
Acknowledgements. Topical Editor T. Pulkkinen thanks D. Sibeck and two other referees for their help in evaluating this paper.

\section{References}

Balogh, A., Carr, C. M., Acuna, M. H., Dunlop, M. W., Beek, T. J., Brown, P., Fornacon, K. -H., Georgescu, E., Glassmeier, K. H., Harris, J., Musmann, G., Oddy, T., and Schwingenschuh, K.: The Cluster magnetic field investigation: overview of in-flight performance and initial results, Ann. Geophys., 19, 1207-1217, 2001 ,

SRef-ID: 1432-0576/ag/2001-19-1207.

Berchem, J. and Russell, C. T.: The thickness of the magnetopause current layer: ISEE 1 and 2 observations, J. Geophys. Res., 87, 2108-2114, 1982.

Bosqued, J.-M., Phan, T. D., Dandouras, I., Escoubet, C. P., Rème, H., Balogh, A., Dunlop, M. W., Alcaydé, D., Amata, E., Bavassano-Cattaneo, M.-B., Carlson, C., DiLellis, A. M. et al.: Cluster Observations of the High-Latitude Magnetopause and Cusp: First Results from the CIS Ion Instruments, Ann. Geophys., Cluster special issue, 19, 1545-1566, 2001.

Bryant, D. A., Krimigis, S. M., and Haerendel, G.: Outline of the Active Magnetospheric Particle Tracer Explorers (AMPTE) mission, IEEE Trans. Geosci. Remote Sensing, GE-23, 177-181, 1985.

Dunlop, M. W., Balogh, A., Glassmeier, K.-H., and the FGM team: Four-Point Cluster Application Of Magnetic Field Analysis Tools: The Curlometer, J. Geophys. Res., 107, 1384-1398, 2002.

Dunlop, M. W., Balogh, A., Glassmeier, K.-H., and the FGM team: Four-Point Cluster Application Of Magnetic Field Analysis Tools: The Discontinuity Analyser, J. Geophys. Res., 107, 1399-1411, 2002.

Elphic, R. C.: Multipoint observations of the magnetopause: results from ISEE and AMPTE, Adv. Space Res., 8, 223-238, 1988.

Elphic, R. C.: Observations of flux transfer events: are FTE's flux ropes, islands, or surface waves?, in Physics of the Magnetic Flux Ropes, Russell, C. T., Priest, E. R., and Lee, L. C. (Eds.), AGU Geophysical Monograph 58, 455-471, 1990.

Elphic, R. C.: Observations of flux transfer events: a review, in Physics of the Magnetopause, Song, P., Sonnerup, B. U. Ö., and Thompsen, M. F. (Eds.), AGU Geophysical Monograph 90, 225233, 1995.

Haaland, S. E., Sonnerup, B., Dunlop, M. W., Balogh, A., Georgescu, E., Hasegawa, H., Klecker, B., Paschmann, G., PuhlQuinn, P., Rème, H., Vaith, H., and Vaivads, A.: Four-spacecraft determination of magnetopause orientation, motion and thickness: comparison with results from single-spacecraft methods, Ann. Geophys., 22, 1347-1365, 2004,

SRef-ID: 1432-0576/ag/2004-22-1347.

Paschmann, G., Papamastorakis, I., Baumjohann, W., Skopke, N., Carlson, C. W., Sonnerup, B. U. Ö., and Lühr, H.: ISEE observations of the magnetopause: Reconnection and the energy balance, J. Geophys. Res., 90, 12 111-12 120, 1985.

Paschmann, G., Papamastorakis, I., Baumjohann, W., Skopke, N., Carlson, C. W., Sonnerup, B. U. Ö., and Lühr, H.: The Magnetopause for large magnetic shear: AMPTE/IRM observations, J. Geophys. Res., 91, 11 099-11 115, 1986.

Phan, T. -D., Paschmann, G., Baumjohann, W., Skopke, N., and Lühr, H.: The magnetosheath region adjacent to the dayside mag- 
netopause: AMPTE/IRM observations, J. Geophys. Res., 99, 121-141, 1994.

Phan, T. -D. and Paschmann, G.: The magnetosheath region adjacent to the dayside magnetopause, in Physics of the Magnetopause, Song, P., Sonnerup, B. U. Ö., and Thompsen, M. F. (Eds.), AGU Geophysical Monograph 90, 115-122, 1995.

Phan, T., Dunlop, M. W., Paschmann, G, Klecker, B., Bosqued, J.-M., Reme, H., Balogh, A., Twitty, C., Mozer, F. S., Carlson, C. W., Mouikis, C., and Kistler, L. M.: Cluster Observations of Continuous Reconnection at the Magnetopause under Steady Interplanetary Magnetic Field Conditions, Ann. Geophys., 22, 2355-2367, 2003,

SRef-ID: 1432-0576/ag/2004-22-2355

Pu, Z. Y., Zong, Q. G. , Fritz, T., Xiao, C. J., Huang, Z. Y., Fu, S. Y., Shi, Q. Q., Dunlop, M. W., Glassmeier, K.-H., Balogh, A., Daly, P., Cao, J.-B., Liu, Z.-X., Rème, H., and Dandouras, J.: Multiple Flux Rope Events at the High-latitude Magnetopause: Cluster/Rapid Observation on 26 January 2001, in press, Surveys of Geophysics, 2005.

Russell, C. T.: The structure of the magnetopause, in Physics of the Magnetopause, Song, P., Sonnerup, B. U. Ö., and Thompsen, M. F. (Eds.), AGU Geophysical Monograph 90, 81-98, 1995.
Russell, C. T. and Elphic, R. C.: Initial ISEE magnetometer results: Magnetopause observations, Space Sci. Rev., 22, 681-715, 1978.

Russell, C. T. and Elphic, R. C.: ISEE observations of flux transfer events at the dayside magnetopause, Geophys. Res. Lett., 6, 33 36, 1979.

Russell, C. T., Mellott, M. M., Smith, E. J., and King, J. H.: Multiple observations of interplanetary shocks: four spacecraft determination of shock normals, J. Geophys. Res., 88, 4739-4748, 1983.

Sibeck, D. G., Lopez, R. E., and Roelof, E. C.: Solar wind control of the magnetopause shape location and motion, J. Geophys. Res., 96, 5489-5495, 1991.

Sonnerup, B. U. Ö. and Cahill, L. J.: Magnetopause structure and attitude from Explorer 12 observations, J. Geophys. Res., 72, 171-183, 1967.

Van Allen, J. A. and Adnan, J.: Observed currents on the Earth's high-latitude magnetopause, J. Geophys. Res., 97, 6381-6395, 1992. 